\title{
A Dynamic Heads-Up Air Traffic Locator \& Collision Advisory Display Using Google Glass
}

\author{
Melvin Rafi ${ }^{1}$, Balaji Chandrasekaran ${ }^{1}$, and Bill Kusmez ${ }^{1}$, \\ Faculty: James E. Steck ${ }^{1}$ and Jibo $\mathrm{He}^{2}$ \\ ${ }^{1}$ Department of Aerospace Engineering, College of Engineering \\ ${ }^{5}$ Department of Psychology, Fairmount College of Liberal Arts and Sciences
}

A heads-up display designed to assist pilots in the rapid location and identification of air traffic is developed and evaluated on Google Glass. The display is conceived as a proposed addition to a Traffic Collision Avoidance System, and the research seeks to determine if the presence of the assistive technology yields faster response times in detecting surrounding air traffic. The display features a dynamically updating three-dimensional arrow that continuously guides the pilot's eye towards oncoming traffic, and updates its orientation based on the relative head motion of the pilot, the motion of the pilot's aircraft, and the location of the oncoming traffic. Testing and simulation runs are conducted to gauge response times of pilots and non-pilots, in an effort to determine if the display leads to faster response times in visually acquiring oncoming traffic. Results show a significant improvement using the display, with reductions of over $60 \%$ in response times. 